\title{
Resting Brains Never Rest: Computational Insights into Potential Cognitive
}

\section{Architectures}

G. Deco ${ }^{(1,2)}$, V.K. Jirsa ${ }^{(3)}$ and AR. McIntosh ${ }^{(4)}$

(1) Center for Brain and Cognition, Computational Neuroscience Group, Department of Information and Communication Technologies, Universitat Pompeu Fabra, Roc Boronat 138, Barcelona, 08018, Spain

(2) Institució Catalana de la Recerca i Estudis Avançats (ICREA), Universitat Pompeu Fabra, Passeig Lluís Companys 23, Barcelona, 08010, Spain

(3) Institut de Neurosciences des Systèmes UMR INSERM 1106, Aix-Marseille Université, Faculté de Médecine, 27, Boulevard Jean Moulin, 13005 Marseille, France

(4) Rotman Research Institute of Baycrest Center, University of Toronto, Toronto, Canada. 


\section{Abstract}

Resting-state networks (RSNs), which have become a large focus in neuroimaging research, can be best simulated by large-scale cortical modelswhen networks teeter on the edge of instability. In this state the functional networks are in a low firing stable state while they are continuously pulled towards multiple other configurations. Small extrinsic perturbations can shape taskrelatednetwork dynamics, while perturbations from intrinsic noise generate excursions reflecting the range of availablefunctional networks. This is distinctly advantageous for the efficiency and speed of network mobilization. Thus, the resting state reflects the dynamical capabilities of the brain, which emphasizes the vital interplay of time and space. We propose a new theoretical framework for RSNsthat can serve as a fertile ground for empirical testing.

\section{Glossary Box}




\section{The Phenomenology of Resting Brains}

The interplay of the brain's intrinsic activity and the external world has seen a revival in the last decade, especially in neuroimaging. A long-held assumption in many of these studies has been that ongoing brain activity is sufficiently random that it averages out in statistical analysis. Hence, imaging studies are termed 'activation' paradigms, where experimental manipulation results in the activation of cerebral circuits that are necessary for performing the task[1,2]. However, despite the most elegant experimental designs, there were consistent patterns of deactivation that often accompany increased cognitive demands. Several researchers began to examine these deactivations based on the idea that the low-level baseline tasks were active states and that the patterns of activation and deactivation represented a shift in the balance from a focus on the subject's internal state and ruminations to the external environment (Andreasen et al., 1995; Nyberg et al., 1996; Shulman et al., 1997). This pattern was later dubbed the "Default-Mode" (Raichle et al., 2001)or more recently the Default Mode Network (DMN).\{Greicius, $2003 \# 4276\}$

The observation that there are relatively consistent distributed patterns of activity during rest has lead to the suggestion that it might be possible to characterize network dynamics without needing an explicit task to drive brain activity. This possibility has been explored in studies of resting-state networks (RSNs)in functional MRI. Probably the first demonstration of a restingstate correlations using fMRI examined the cross-correlation (that is, functional connectivity) between activity in the primary motor cortex (M1) and other brain regions independent of any overt task (Biswal et al., 1995). Spatially, the functional connectivity pattern seemed to mimic the pattern of activation seen when subjects executed an overt motor response. This observation led to a veritable explosion of work exclusively focused on the identification and 
characterization of these networks (Greicius et al., 2003; Lowe et al., 1998; Rogers et al., 2007). More recently, RSN have been studied with magnetoencephalography (MEG) and electroencephalography (EEG) (add refs). In general, EEG and MEG studies of resting-state activity have found slow fluctuations in the power of alpha and beta-frequency oscillations, which correlate across distant brain areas. Notably, these band-limited power (BLP) fluctuations yield large-scale spatial maps, some of which correspond quantitatively to the resting-state networks derived from fMRI (10). Overall, these results indicate that resting state functional connectivity in BOLD responses corresponds to a spatially structured modulation of BLP fluctuations.

Much of the RSN work in neuroimaging has emphasized the consistency of the spatial pattern, but that is likely a reflection of the relatively long time course over which the functional connectivity was estimated. RSNs have a rich spatiotemporal signature. The first clue to this behavior came from large-scale network simulations using anatomically-realistic cortical connectivity(Honey et al., 2007). Across a long time window, distinct functional networks formed that relate to the structural connnections. At shorter time steps, however, subnetworks form and dissolve as the full network evolves. The regions within each of the broad spatial patterns would move away from their core network to form other networks, and then transition to a new set of spatial patterns. A schematic of this behavior is shown in Figure 1, where a 'core' pattern of functional connectivity is identified (Figure 1a), for example by looking at the correlation across a long-time window, but when smaller time windows are considered (Figure 1b), the core pattern dissolves and re-emerges. This temporal dependency has been empirically validated in fMRI studies (Chang and Glover, 2010, \{Allen, $2012 \# 5678$ \}, and emphasizes the rich spatiotemporal dynamics of resting-state brain networks. 


\section{Neurodynamical Mechanisms underlying the Resting State}

Spatio-temporal functional connectivity in RSNs was originally hypothesized to reflect the underlying anatomical connectivity structure. If we assume that each independent local cortical area shows low level noisy neural ongoing activity, i.e. Poissonian spiking activity around 3-10 Hz, as evidenced experimentally with neuronal recordings(Burns and Webb, 1976; Koch and Fuster, 1989; Wilson et al., 1994), then the spatial structure of the underlying anatomical coupling between those brain areas would support correlations of the noisy fluctuations. Indeed the correlation structure of spontaneous BOLD fluctuations relates to the underlying anatomical circuitry in monkey(Vincent et al., 2007). In particular, there is a strong similarity between retrograde tracer maps reflecting anatomical connectivity and the resting BOLD functional connectivity. Furthermore, the networks of coherent spontaneous BOLD fluctuations under anesthesia are similar to networks commonly engaged during task performance in awake animals.

In spite of the relationship of anatomical and functional connectivity, the link is not perfect. Areas can show functional connectivity without a direct anatomical link and likely mediated through a series of indirect links\{Honey, $2010 \# 5686$;Shen, 2012 \#5469\}and, as noted above, also very dependent on the time window over which functional connectivity is computed. Thus, while RSNs may depend on the anatomical connectivity they cannot be understood in those terms alone (Deco et al., 2009; Deco et al., 2011; Ghosh et al., 2008b). The missing link for understanding the formation and dissolution of resting state networks is the dynamics (Deco et al., 2011). Different local brain areas show specific dynamics; how these different dynamics 
interact through the anatomical coupling is not intuitive. Thus, theoretical models that bind structure and dynamics are fundamental for studying the relation between anatomical structure and RSN dynamics and explicitly show that structure shapes functional connectivity but is definitively not identical to that(Cabral et al., 2011; Honey et al., 2007; Honey et al., 2009; Knock et al., 2009). Models of resting state activity have three ingredients: (i) the underlying anatomical connectivity,(ii) the time delays due to signal transmission across different distances and (iii) the local brain area dynamics (see Figure 2). The main theoretical goal is to study,the critical dynamical features of the model that are able to fit RSNs in order to understand the mechanisms that explain their origin.

The first ingredient of these models is of course the anatomical structure. In all models, the structural information is extracted from databases compiling different type of tracing studies (e.g. for the macaque cortex this information is provided by the CoCoMac neuroinformatics (Kotter, 2004), or from DTI/DSI (Diffusion Tensor Imaging/ Diffusion Spectrum Imaging) techniques (Hagmann et al., 2008). The second ingredient is the signal transmission time delays. Generally time delays do not alter the stationary spatial patterns in a network, but they do change their stability (Campbell, 2007). Though time delays play an essential role in the synchronization behaviour of oscillatory patterns, it is less clear to what extent they influence patterns of spiking neurons. Various RSN models have demonstrated that the space-time structure of the couplings shapes the emergent network dynamics (Cabral et al., 2011; Deco et al., 2009; Ghosh et al., 2008a; Ghosh et al., 2008b), though RSN models based on spiking neurons ignored the time delays due to the absence of oscillations (Deco and Jirsa, 2012). The third ingredient of the models is the type of intrinsic dynamics of the local nodes. Models considered simple oscillatory dynamics(Cabral et al., 2011; Deco et al., 2009; Ghosh et al., 
2008a; Ghosh et al., 2008b), chaotic dynamics(Honey et al., 2007), and finally, very detailed realistic local networks with excitatory and inhibitory populations of spiking neurons coupled through N-Methyl-D-aspartic acid (NMDA),alpha-amino-3-hydroxy-5-methylisoxazole-4propionic acid (AMPA) and gamma-Aminobutyric acid (GABA) synaptic dynamics(Deco and Jirsa, 2012).

A common characteristic of all models is that the optimal working point for explaining the emergence of RSN is at the edge of instability, the critical point of a bifurcation. The first account ofthe RSN dynamics arising under conditions of criticality (Ghosh et al., 2008b)demonstrated that the resting state emerges from noise-induced transient fluctuations around the stable equilibrium state of a network of coupled FitzHugh-Nagumo oscillators just below the edge of instability. The noise provokes fluctuations between different multi-stable oscillatory brain states in a network of Wilson-Cowan oscillators, again in the neighborhood of the critical point, but above the edge of instability(Deco et al., 2009). RSNs also result from instability due to chaotic fluctuations(Honey et al., 2007). In other words, the type of local dynamics is relevant for determining whether that particular dynamics shows the relevant criticality, such that working at that point generates the resting functional connectivity. Nevertheless, in all models, the underlying anatomical structure shapes the structure of the dynamical landscape, which then the fluctuations of the network model can explore. The time delays then may alter the location of the instability, but will not change the actual structure of spatial patterns in the dynamical landscape. This is the reason why, generally, all the models could explain the spatial functional correlations defining the different RSNs. At the edge of the critical instability of any model the spatial correlations of the noisy excursions are mainly shaped by the anatomical structure. The degree to which the RSNs are expressed depends on the proximity to the instability, which is 
determined by the space-time structure of the couplings, that is the topology of the connectivity and its associated time delays. In other words, by working near instability, RSNs reflect the dynamical capacity of that system to take on different functional networks. The global dynamics of a brain working at a critical point amplify the underlying structure of the anatomical connections and of its interactions with the local dynamics. Importantly, if the system was not working at that critical point, then the noisy fluctuations would not reflect any structure whatsoever, the RSNs would not exist and the resting state would be uncorrelated noise.

Hypotheses related to criticality and brain function have been put forward previously; for instance one example(Haken, 1996)posits that the brain operates in the close proximity of socalled saddle points, which are characterized by stable and unstable directions in the brain's state space. As the brain network dynamics evolves, various saddle points are visited giving rise to the expression of brain states, because the dwell times at these points are significantly longer than anywhere else. These states might be related to cognitive processes, which is reminiscent of another proposal (Rabinovich et al., 2001; Rabinovich et al., 2008) that heteroclinic cycles are composed of chains of cognitively relevant brain states. The working point of neural networks at criticality has also been noted in the context of optimized information processing. Simulations predicted that at this point of criticality, neuronal networks optimize several aspects of information processing, (Kinouchi and Copelli, 2006; Beggs and Plenz, 2003; Tanaka et al., 2009). Experimental support of these predictions has been provided by demonstrating that in vitro cortical networks have maximum dynamic range when spontaneous activity takesthe form of "neuronal avalanches" (Shew et al JNS 2009). During rest in the awake monkey, the avalanches constitute the dominant form of ongoing cortical activity (Petermann et al., 2009). 
For the RSN dynamics, the discussion of the potential role of criticality as an organizing principle has been further explored with a realistic spiking cortical network that was microscopically organized as standard attractor models (known from neural models of memory, attention, decision making, etc. (Deco and Rolls, 2006; Deco et al., 2008)) and macroscopically organized through a large-scale anatomical connection matrix obtained from human subjects via DTI/DSI tractography (Hagmann et al., 2008) (Deco and Jirsa 2012). The model of a local brain area consists of integrate-and-fire spiking neurons with excitatory (AMPA and NMDA) and inhibitory (GABA-A) synaptic receptor types. Figure 3 shows the main results and explains the mechanisms causing resting state activity. Let us assume that the control parameter is the coupling strength different brain areas. The global dynamical states of the system can be described by a so-called bifurcation diagram, which captures the stationary states (attractors) of the system as a function of the coupling strength. For low coupling strength, only a stable spontaneous state, characterized by an asynchronous low level firing activity in all brain areas. By increasing the coupling strength at the first bifurcation point, new stable attractors emerge. These new attractors reflect increased activity in some brain areas, defining the emergence and stabilization of specific networks of brain areas. In Figure 3, this is shown in the landscape cartoon (Figure $3 b)$, and in the bifurcation diagram on top of that, where the maximal rate activity in the brain is plotted. After the first bifurcation a new branch appears evidencing the emergenceof new attractors. Nevertheless, after this first bifurcation, the spontaneous state is still stable. Only after a second bifurcation the spontaneous state loses stability. It is precisely at the edge of this second bifurcation point, where the brain seems to operate. In the top panel, we show the fitting between the empirical and simulated resting BOLD functional connectivity. The best fitting (maximal correlation) occurs exactly at the edge of the second bifurcation, where the 
spontaneous state loses its stability and therefore the noisy fluctuations of the dynamics are able to explore and reflect the structure of the other "cognitive" attractors shaped by the underlying anatomy. While this constitutes the origin and explanation of the resting state, it does not explain why the brain operates at that particular bifurcation. The right panel of Figure 3 provides the answer. The critical point is an optimal working point from an economical and ecological point of view. At that particular point, the system is maximally sensitive to external stimulations and able to respond fast and efficiently even to weak "gentle" stimulations. In the figure, the visual network consisting of 13 brain areas is stimulated by the application of an external bias in the V1 area and the convergence to the appropriate network attractor for different strengths of the external stimulating bias was measured. Figure $3 c$ shows the convergence characteristics for two different working points, one at the edge of the second bifurcation and one further away. The most optimal working point is indeed the one observed experimentally, i.e. at the brink of the second bifurcation, because it offers an advantage in terms of response time and sensitivity to incoming information.

\section{Conclusions and outlook}

As brain network properties change, be it due to inter subject variability, learning, disease, ageing or development, the critical point of the network will change and consequently its associated dynamic features including the subspace spanned by the resting state networks and optimality of information processing through the network. If criticality is indeed a principle of functional brain organization, then homeostatic mechanisms are required to maintain the brain network at criticality, which can be explored in theoretical and empirical work (e.g, Box 1).

Consideration of criticality as a principle of functional brain organization has implications for the emergence of cognitive processes in the context of RSN's . Rather than emboding a specific 
cognitive or behavioural operations, RSN dynamics are the gateway to the array of cognitive architectures the brain has available (Box 2). From this perspective, RSN dynamics represent the instigation of lower-dimensional subspaces within the available high-dimensional space. The lower-dimensional subspaces define a specific workspace that constitutes some sort of prospective exploration of potential functions, but not their instantiation. In a sense, RSN's represent the brain's exploration of what is possible

\section{Box 1. Outstanding issues \& questions}

Can we measure the critical point empirically?: To address this question we need to measure the critical point directly from empirical studies. The mixing of deterministic and stochastic contributions of the network dynamics renders this task difficult, but not impossible. The variability of the brain signals will typically scale with the ratio $Q / L$, in which $L$ denotes the linear stability strength of the equilibrium attractor and $Q$ the noise strength. The disentangling of deterministic and noise contributions is possible by measuring statistical quantifiers including the variance of the brain signals, the dwell time at individual attractors, as well as the mean escape time of resting state attractors(e.g., Schoner et al., 1986).

Can we manipulate the critical point?: exogenous drives such as transcranial magnetic 
stimulation or Deep Brain Stimulation offer independent means of testing our understanding of the RSN dynamics. In particular, criticality predicts that the RSNs are closer to instability than other networks as quantified by the linear stability strength L. The networks closest to instability (with the smallest $\mathrm{L}$ ) will determine the overall brain dynamics after a perturbation and show the longest relaxation times (scaling inversely to $\mathrm{L}$ ) returning towards equilibrium.

Box 2. -Hypotheses about Resting-state dynamics and cognitive potential-

i) The potential should increase with maturation. This was confirmed in EEG and MEG studies of infants and children up to $15 \mathrm{yrs}$, where the entropy increased with age. Importantly, the greater entropy, and more specifically multiscale entropy, correlated with higher cognitive performance(Lippe et al., 2009; McIntosh et al., 2008; Misic et al., 2010). Also of interest was the observation that this increase in entropy could be attributed to high exchange of information between distal brain regions, rather than an increase of local dynamics (Vakorin et al., 2011).

ii) The potential should change in senescence. Some recent works examine variability in BOLD $\mathrm{fMRI}$ across different aged adults and show a general decrease in dynamics, though on a much less broad range than in early maturation \{Garrett, 2010 \#4245;Garrett, 2011 \#4959;Garrett, 2012 \#4957;McIntosh, 2013 \#5465\}. This is consistent with the fact that the cognitive changes in aging, while significant, are not nearly as striking as in early maturation.

iii) The potential should be affected by brain disease and disorders. Studies of dementia clearly show reduced MSE with disease severity (Takahashi et al., 2009), while 
studies of recovery of function from head injury show increased dynamics linked to better recovery (Nenadovic et al., 2008; Raja Beharelle et al., 2012)

iv) The potential should increase with learning. Recent work with EEG demonstrated that as persons acquire more knowledge about an object, the dynamics elicited by that object (i.e., the number of potential network configurations) is also increased (Heisz et al., 2012)

\section{GLOSSARY BOX:}

\section{Activation Paradigm}

Experimental design in which the subject is asked to execute a perceptual, motor or cognitive task. During task execution the associated brain activation is measured and considered the only neural correlate of the specific function.

\section{Attractor Networks}

Brain dynamics can be modeled by attractor network models. They consist of a network of neurons which is a dynamical system that in general has the tendency to settle in stationary states, fixed points called "attractors", typically characterized by a stable pattern of firing activity. External or even intrinsic noise that appears in the form of finite size effects could provoke destabilization of an attractor inducing therefore transitions between different stable attractors. The dynamics of the network can be described by coupling the dynamical equations describing each neuron and the synaptic variables associated with their mutual coupling.

\section{Bifurcation}

Is one of the basic tools of analysis of dynamical systems. A bifurcation is defined by qualitative changes of the asymptotic behavior of the system ("attractors") under parameter variation.

\section{Chaos}

Behavior of a dynamical system that is highly sensitive to initial conditions in such a way that extremely small differences in initial conditions yield widely diverging outcomes so that the evolution of the system is effectively unpredictable, even if the system is purely deterministic.

\section{Criticality}

At the brink of a bifurcation, the system displays certain characteristic dynamic features, of which most are related to enhanced fluctuations.

\section{Diffusion spectrum imaging (DSI)}

An MRI technique that is similar to DTI, but with the added capability of resolving multiple directions of diffusion in each voxel of white matter. This allows multiple groups of fibres at each location, including intersecting fibre pathways, to be mapped.

\section{Diffusion tensor imaging (DTI)}


AnMRI technique that takes advantage of the restricted diffusion of water throughmyelinated nerve fibres in thebrain to infer the anatomical connectivity between brain areas.

\section{Functional connectivity}

The relationship between activity in two or more neural sources. This usually refers to the temporal correlation between sources but has been extended to include correlations across trials or different experimental subjects. Functional connectivity methods include estimation of correlation coefficients and coherence. The estimation cannot be used to infer the direction of the relation between sources.

\section{Mean-Field}

The mean-field approximation consists of replacing the temporally averaged discharge rate of a cell with an equivalent momentary activity of a neural population (ensemble average) that corresponds to the assumption of ergodicity. According to this approximation, we characterize each cell assembly by means of its activity population rate.

Neural avalanches: Bursts of elevated population activity, correlated in space and time, that are distinguished by a particular statistical character: activity clusters of size s occur with probability $\mathrm{P}(\mathrm{s})$ equal to s-a i.e., a power law with exponent $\mathrm{a}=1.5$.

Noise

In neurodynamical systems, noise is mainly given by the probabilistic spiking times of neurons and usually plays an important and advantageous role in brain function. Spiking noise is a significant factor in a network with a finite (i.e., limited) number of neurons. The spiking noise can be described as introducing statistical fluctuations into the finite-size system.

\section{Spiking Dynamics}

A network of spiking neurons establishes a high dimension dynamical system in which individual neurons (usually expressed by Integrate-and-Fire or Hodgkin-Huxley models) interact with each other thorough different type of dynamical synapses (e.g. AMPA, NMDA, GABA).

\section{Wilson-Cowan Oscillators}

Mean-field-like rate model expressing the coupling between two populations of excitatory and inhibitory neurons. In general, the Wilson-Cowan model is tuned such that the population rate of the pools oscillates. It is one of the simple neural oscillators. 


\section{$\underline{\text { References }}$}

[1] Petersen, S.E., Fiez, J.A., 1993. The processing of single words studied with positron emission tomography. Annual Review of Neuroscience. 16.

[2] Posner, M.I., Petersen, S.E., Fox, P.T., Raichle, M.E., 1988. Localization of cognitive operations in the human brain. Science. 240, 1627-1631.

Allen, E., Damaraju, E., Plis, S., Erhardt, E., Eichele, T., Calhoun, V., in press. Tracking whole-brain connectivity dynamics in the resting-state. Cerebral Cortex.

Andreasen, N.C., O'Leary, D.S., Cizadlo, T., Arndt, S., Rezai, K., Watkins, G.L., Boles Ponto, L.L., Hichwa, R.D., 1995. Remembering the past: Two facets of episodic memory explored with positron emission tomography. Am. J. Psychiatr. 152, 1576-1585.

Biswal, B., Yetkin, F.Z., Haughton, V.M., Hyde, J.S., 1995. Functional connectivity in the motor cortex of resting human brain using echo-planar MRI. Magn Reson Med. 34, 537-41.

Burns, B.D., Webb, A.C., 1976. The spontaneous activity of neurones in the cat's cerebral cortex. Proceedings of the Royal Society of London. Series B, Containing papers of a Biological character. Royal Society. 194, 211-23.

Cabral, J., Hugues, E., Sporns, O., Deco, G., 2011. Role of local network oscillations in restingstate functional connectivity. Neuroimage. 57, 130-9.

Campbell, S.A., 2007. Time delays in neural systems. In: Handbook of Brain Connectivity. Vol., V.J. Jirsa, A.R. Mclntosh, ed.^eds. Springer, Berlin, pp. 65-90.

Chang, C., Glover, G.H., 2010. Time-frequency dynamics of resting-state brain connectivity measured with fMRI. Neuroimage. 50, 81-98.

Damoiseaux, J.S., Rombouts, S.A., Barkhof, F., Scheltens, P., Stam, C.J., Smith, S.M., Beckmann, C.F., 2006. Consistent resting-state networks across healthy subjects. Proc Natl Acad Sci U S A. 103, 13848-53.

Deco, G., Rolls, E.T., 2006. Decision-making and Weber's law: a neurophysiological model. Eur J Neurosci. 24, 901-16.

Deco, G., Jirsa, V.K., Robinson, P.A., Breakspear, M., Friston, K., 2008. The dynamic brain: from spiking neurons to neural masses and cortical fields. PLoS computational biology. 4, e1000092.

Deco, G., Jirsa, V., McIntosh, A.R., Sporns, O., Kotter, R., 2009. Key role of coupling, delay, and noise in resting brain fluctuations. Proc Natl Acad Sci U S A. 106, 10302-7.

Deco, G., Jirsa, V.K., McIntosh, A.R., 2011. Emerging concepts for the dynamical organization of resting-state activity in the brain. Nature reviews. Neuroscience. 12, 43-56.

Deco, G., Jirsa, V.K., 2012. Ongoing cortical activity at rest: criticality, multistability, and ghost attractors. Journal of neuroscience. 32, 3366-75.

Garrett, D.D., Kovacevic, N., McIntosh, A.R., Grady, C.L., 2010. Blood oxygen level-dependent signal variability is more than just noise. J Neurosci. 30, 4914-21.

Garrett, D.D., Kovacevic, N., McIntosh, A.R., Grady, C.L., 2011. The importance of being variable. The Journal of neuroscience : the official journal of the Society for Neuroscience. 31, 4496-503.

Garrett, D.D., Kovacevic, N., McIntosh, A.R., Grady, C.L., 2012. The Modulation of BOLD Variability between Cognitive States Varies by Age and Processing Speed. Cerebral Cortex.

Ghosh, A., Rho, Y., McIntosh, A.R., Kotter, R., Jirsa, V.K., 2008a. Cortical network dynamics with time delays reveals functional connectivity in the resting brain. Cogn Neurodyn. 2, 115- 
20.

Ghosh, A., Rho, Y., McIntosh, A.R., Kotter, R., Jirsa, V.K., 2008b. Noise during Rest Enables the Exploration of the Brain's Dynamic Repertoire. PLoS Computational Biology. 4, e1000196.

Greicius, M.D., Krasnow, B., Reiss, A.L., Menon, V., 2003. Functional connectivity in the resting brain: a network analysis of the default mode hypothesis. Proc Natl Acad Sci U S A. 100, 253-8.

Hagmann, P., Cammoun, L., Gigandet, X., Meuli, R., Honey, C.J., Wedeen, V.J., Sporns, O., 2008. Mapping the structural core of human cerebral cortex. PLoS Biol. 6, e159.

Haken, H., 1996. Principles of Brain Functioning: A synergetic approach to brain activity, behavior and cognition, Vol., Springer, Berlin.

Heisz, J.J., Shedden, J.M., Mclntosh, A.R., 2012. Relating brain signal variability to knowledge representation. Neuroimage. 63, 1384-92.

Honey, C.J., Kotter, R., Breakspear, M., Sporns, O., 2007. Network structure of cerebral cortex shapes functional connectivity on multiple time scales. Proc Natl Acad Sci U S A. 104, 10240-5.

Honey, C.J., Sporns, O., Cammoun, L., Gigandet, X., Thiran, J.P., Meuli, R., Hagmann, P., 2009. Predicting human resting-state functional connectivity from structural connectivity. Proc Natl Acad Sci U S A. 106, 2035-40.

Knock, S.A., Mclntosh, A.R., Sporns, O., Kotter, R., Hagmann, P., Jirsa, V.K., 2009. The effects of physiologically plausible connectivity structure on local and global dynamics in large scale brain models. J Neurosci Methods. 183, 86-94.

Koch, K.W., Fuster, J.M., 1989. Unit activity in monkey parietal cortex related to haptic perception and temporary memory. Experimental brain research. Experimentelle Hirnforschung. Experimentation cerebrale. 76, 292-306.

Kotter, R., 2004. Online retrieval, processing, and visualization of primate connectivity data from the CoCoMac database. Neuroinformatics. 2, 127-144.

Lippe, S., Kovacevic, N., McIntosh, A.R., 2009. Differential maturation of brain signal complexity in the human auditory and visual system. Front Hum Neurosci. 3, 48.

Lowe, M.J., Mock, B.J., Sorenson, J.A., 1998. Functional connectivity in single and multislice echoplanar imaging using resting-state fluctuations. Neuroimage. 7, 119-32.

McIntosh, A.R., Kovacevic, N., Itier, R.J., 2008. Increased brain signal variability accompanies lower behavioral variability in development. PLoS Comput Biol. 4, e1000106.

Misic, B., Mills, T., Taylor, M.J., McIntosh, A.R., 2010. Brain noise is task dependent and region specific. Journal of Neurophysiology. 104, 2667-76.

Nenadovic, V., Hutchison, J.S., Dominguez, L.G., Otsubo, H., Gray, M.P., Sharma, R., Belkas, J., Perez Velazquez, J.L., 2008. Fluctuations in cortical synchronization in pediatric traumatic brain injury. J Neurotrauma. 25, 615-27.

Nyberg, L., McIntosh, A.R., Cabeza, R., Nilsson, L.-G., Houle, S., Habib, R., Tulving, E., 1996. Network analysis of positron emission tomography regional cerebral blood flow data: Ensemble inhibition during episodic memory retrieval. Journal of Neuroscience. 16, 3753-3759.

Rabinovich, M., Volkovskii, A., Lecanda, P., Huerta, R., Abarbanel, H.D., Laurent, G., 2001. Dynamical encoding by networks of competing neuron groups: winnerless competition. Physical Review Letters. 87, 068102.

Rabinovich, M.I., Huerta, R., Varona, P., Afraimovich, V.S., 2008. Transient cognitive dynamics, metastability, and decision making. PLoS computational biology. 4, e1000072.

Raichle, M.E., MacLeod, A.M., Snyder, A.Z., Powers, W.J., Gusnard, D.A., Shulman, G.L., 2001. A 
default mode of brain function. Proc Natl Acad Sci U S A. 98, 676-82.

Raja Beharelle, A., Kovacevic, N., McIntosh, A.R., Levine, B., 2012. Brain signal variability relates to stability of behavior after recovery from diffuse brain injury. Neuroimage. 60, 152837.

Rogers, B.P., Morgan, V.L., Newton, A.T., Gore, J.C., 2007. Assessing functional connectivity in the human brain by fMRI. Magn Reson Imaging. 25, 1347-57.

Schoner, G., Haken, H., Kelso, J.A., 1986. A stochastic theory of phase transitions in human hand movement. Biological Cybernetics. 53, 247-57.

Shulman, G.L., Fiez, J.A., Corbetta, M., Buckner, R.L., Miezin, F.M., Raichle, M.E., Petersen, S.E., 1997. Common Blood Flow Changes across Visual Tasks: II. Decreases in Cerebral Cortex. Journal of Cognitive Neuroscience. 9, 648-663.

Takahashi, T., Cho, R.Y., Murata, T., Mizuno, T., Kikuchi, M., Mizukami, K., Kosaka, H., Takahashi, K., Wada, Y., 2009. Age-related variation in EEG complexity to photic stimulation: a multiscale entropy analysis. Clinical neurophysiology : official journal of the International Federation of Clinical Neurophysiology. 120, 476-83.

Vakorin, V.A., Lippe, S., McIntosh, A.R., 2011. Variability of brain signals processed locally transforms into higher connectivity with brain development. The Journal of neuroscience : the official journal of the Society for Neuroscience. 31, 6405-13.

Vincent, J.L., Patel, G.H., Fox, M.D., Snyder, A.Z., Baker, J.T., Van Essen, D.C., Zempel, J.M., Snyder, L.H., Corbetta, M., Raichle, M.E., 2007. Intrinsic functional architecture in the anaesthetized monkey brain. Nature. 447, 83-6.

Wilson, F.A., O'Scalaidhe, S.P., Goldman-Rakic, P.S., 1994. Functional synergism between putative gamma-aminobutyrate-containing neurons and pyramidal neurons in prefrontal cortex. Proceedings of the National Academy of Sciences of the United States of America. 91, 4009-13. 


\section{Figure Captions}

Figure 1. Schematic of the transitions of functional connectivity in different resting state networks across time.a) The left most panel depicts a correlation matrix (functional connectivity) among network nodes across a long time interval. In fMRI BOLD studies, this can be as long as 10 minutes. The clustering in the matrix indicates 3 networks dominant at that time window. b)The change in functional connectivity of the nodes at shorter time window. Here the dominant pattern repeats (e.g., times T1 and T4), but different subnetworks form as the system 'explores' different functional architectures. This behavior of resting state networks has been shown both in simulations (Honey et al., 2007) and in empirical work (Allen et al., in press).

Figure 2. Neuroanatomical connectivity data obtained by DSI and tractography are used for defining the structural connectivity between the different brain areas. Indeed, for a given parcellation a neuroanatomical structural connectivity matrix $\mathrm{C}$ links the $\mathrm{N}$-parcellated cortical regions with clear anatomical landmarks. A neurodynamical model is then constructed using a set of stochastic differential equations coupled according to the connectivity matrix $\mathrm{C}$. The model is then constrained by fitting the functional connectivity observed in empirical data. In our case, the empirical functional connectivity was measured using fMRI BOLD activity. This framework enables us to study the link between anatomical structure and resting-state dynamics. 
Figure 3. The large-scale dynamical brain model is able to best fit the empirical resting functional MRI data when the brain network is critical, i.e. at the border of a dynamical bifurcation point, so that at that operating point the system defines a meaningful dynamic repertoire that is inherent to the neuroanatomical connectivity.a) To determine the dynamical operating point of the system, we contrasted the results of the simulated model with the experimental resting FC as a function of the control parameter $\mathrm{G}$ describing the scaling or global strength of the inter-cortical coupling. The fit between both the empirical and the simulated FC matrix is measured by the Pearson correlation coefficient. In the same plot, the second bifurcation line obtained below is also shown. The best fit of the empirical data is observed at the brink of the second bifurcation models. b) Bifurcation diagrams characterizing the stationary states of the brain system as a function of the control parameter G. We plot for the different possible stable states, the maximal firing rate activity over all cortical area. We studied 1000 different random initial conditions in order to identify all possible new stationary states, and also the case where the initial condition is just the spontaneous state, in order to identify when the spontaneous state losses stability. For small values of the global coupling $G$, only one stable state exists, namely the spontaneous state characterized by low firing activity in all cortical areas. For a critical value of $\mathrm{G}$ a first bifurcation emerges where at least one new multi-stable state appears while the spontaneous state is still stable. For even larger values of $\mathrm{G}$, a second bifurcation appears where the spontaneous state becomes unstable. c) Cartoon of the corresponding attractor landscape. Left column: The critical point, where the trivial spontaneous state loses stability is an optimal working point from an economical and ecological point of view. At that particular point the system is maximally sensitive to external stimulations, i.e. is able to respond fast and efficiently even to weak "gentle" stimulations. The figure plots how fast the system converges to the appropriate network attractor as a function of the strength of the 
external stimulating bias. In particular, we show the convergence characteristics for two different working points, one at the edge of the second bifurcation and one further away. The most optimal working point is indeed the one observed experimentally, i.e. at the brink of the second bifurcation, because it offers an advantage in terms of response time and sensitivity to incoming information. 